\title{
Sodium enrichment of stellar atmospheres
}

\section{Non-variable supergiants and bright giants}

\author{
S. M. Andrievsky ${ }^{1,2,3}$, I. A. Egorova ${ }^{1,3}$, S. A. Korotin ${ }^{1,3}$, and R. Burnage ${ }^{4}$ \\ 1 Department of Astronomy, Odessa State University, Shevchenko Park, 65014 Odessa, Ukraine \\ e-mail: serkor@skyline.od.ua; egorova@deneb.odessa.ua \\ 2 Instituto Astronômico e Geofísico, Universidade de São Paulo, Av. Miguel Stefano, 4200 Agua Funda, Brasil \\ 3 Odessa Astronomical Observatory and Isaac Newton Institute of Chile, Odessa Branch, Ukraine \\ 4 Observatoire de Haute-Provence, 04870 Saint-Michel l'Observatoire, France
}

Received 24 December 2001 / Accepted 15 April 2002

\begin{abstract}
. 48 supergiants and bright giants have been observed in order to investigate the sodium enrichment of their atmospheres and its connection with stellar gravity.

We present the equivalent widths of the $6154 \AA$ and $6160 \AA$ NaI lines measured from the program spectra, the results of effective temperature determinations, the NLTE sodium abundances, and the derived relation between the sodium overabundance and surface gravity.
\end{abstract}

Key words. stars: abundances - stars: supergiants

\section{Introduction}

One of the long-standing problems in observational stellar astrophysics is the sodium enrichment of supergiant atmospheres. First systematic attempt to find a relationship between the sodium overabundance and stellar mass was undertaken by Sasselov (1986), who used rather non-homogeneous literature data on sodium abundance $[\mathrm{Na} / \mathrm{H}]$ for 34 stars. He found that the sodium abundance has a tendency to increase with increasing mass, but quantitative characteristics of that relation were probably significantly affected by the non-homogeneity of the $[\mathrm{Na} / \mathrm{H}]$ values and somewhat unreliable mass determination.

From theoretical point of view, this problem was investigated by Denissenkov (1990), Prantzos et al. (1991) and El Eid \& Prantzos (1993). These authors, using the sodium abundances in $\mathrm{F}$ supergiants, overestimated by Boyarchuk et al. (1988), concluded that there is a necessity either to increase the initial abundance of ${ }^{22} \mathrm{Ne}$ (that, in principle, cannot be observationally checked for the supergiants), or to increase the rate of the ${ }^{20} \mathrm{Ne}(\mathrm{p}, \gamma)^{21} \mathrm{Na}$

Send offprint requests to: S. M. Andrievsky, e-mail: scan@deneb.odessa.ua reaction in order to achieve match between the observations and theory predictions.

Later, El Eid \& Champagne (1995) criticized those first attempts, and re-investigated the sodiun enrichment in A-F type supergiants using the data on $\mathrm{Na}$ excess provided by Takeda \& Takada-Hidai (1994). Those authors considered the nucleosynthesis of ${ }^{23} \mathrm{Na}$ in the stars with masses 5-19 $M_{\odot}$. A positive correlation between $[\mathrm{Na} / \mathrm{H}]$ and mass was found. Nevertheless, inspecting Fig. 3 from that work, one can conclude that the correlation between observed and calculated sodium overabundance is not well established (mainly due to the uncertainties in the observed sodium abundances).

Fragmentary observational data on $[\mathrm{Na} / \mathrm{H}]$ in galactic supergiants can be found in numerous publications, but usually they are obtained using different methods, oscillator strengths and atmosphere models, spectral material of different quality and different sodium lines (in some cases using only strong lines with $W_{\lambda} \geq 200 \mathrm{~m} \AA$, that are not appropriate for LTE analysis).

In this work we present the results of a homogeneous spectroscopic investigation of a sample of $\mathrm{F}-\mathrm{G}$ supergiant and bright giant stars based on LTE and NLTE calculations. 


\section{Observations}

The observations of 48 program supergiants and bright giants of the $\mathrm{F}-\mathrm{G}$ spectral classes were done at the $1.52-\mathrm{m}$ telescope of the Observatoire de Haute Provence (23 July to 30 July 1998). Thompson TH 7832 LA detector consisting of 2048 photodiodes with the AURELLIE spectrograph (1200 lines $\mathrm{mm}^{-1}$ grating in the second order) was used. The spectral domain was centered on the wavelength $6150 \AA$ and covered the region from $6050 \AA$ to $6250 \AA$. This spectral region has been selected in order to observe two sodium lines, $6154 \AA$ and $6160 \AA$, that are usually not very strong in the supergiant spectra and can be used for the LTE analysis. The resolving power was 25000 . For the great majority of the program spectra the $S / N$ ratio varied from 200 to 400 .

A preliminary reduction of the spectroscopic data (an extraction from the images and wavelength calibration) was made using the MIDAS package. Further steps of the analysis (continuum level placement and equivalent width determinations) were performed with the help of DECH20 code (Galazutdinov 1992). The equivalent widths of selected sodium lines were measured in the gaussian approximation (see Table 2 , the equivalent widths are given in $\mathrm{m} \AA$ ).

\section{Atmospheric parameters}

\subsection{The temperature estimation}

We used photometric data for program stars to estimate their effective temperatures $T_{\text {eff. }}$. All the necessary information about colour indices was selected from the SIMBAD database. From the individual estimates of colour indices that are available for each star, we derived the mean values. When it was possible, the reddenings were taken from the literature. To transform $E(B-V)$ or $E(b-y)$ values into $E(B 2-V 1), E(R-I)$ and $E(V-R)$, we used the following expressions:

$$
\begin{aligned}
& E(b-y)=0.73 \times E(B-V) \\
& E(B 2-V 1)=0.83 \times E(B-V) \\
& E(R-I)=0.62 \times E(B-V) \\
& E(V-R)=0.92 \times E(B-V) .
\end{aligned}
$$

In some cases, when the reddenings were not available, we calculated $E(B-V)$ values using the colour indices and formula given by Arellano Ferro \& Parrao (1990).

To estimate the temperatures we used several calibrating relations: $T(b-y)-$ Kurucz (1991) calibration; $T(B-V)-$ Kurucz (1991); $T(R-I)-$ Buser \& Kurucz (1992); $T(V 1-B 2)-$ Kunzli et al. (1997); $T(V-R)-$ Castelli et al. (1997) calibration.

When photometric data were not available, the effective temperature was estimated by avoiding any visible dependence between the iron abundance from Fe I lines and the excitation potentials of their lower level. Obtained results are given in Table 1 . As one can see, the different calibrations give close estimates of the effective temperatures.

\subsection{Gravity and microturbulent velocity}

To estimate the microturbulent velocities and gravities we used the usual method based on avoiding any dependence of iron abundance derived from the Fe I lines and their equivalent widths, and subsequent adjustment of iron abundance from FeI and Fe II lines. It should be noted that such an approach has been criticized by Kovtyukh \& Andrievsky (1999) as producing somewhat incorrect gravities, but in our case there was no possibility of using the Fe II lines for the determination of atmospheric parameters (as proposed by Kovtyukh \& Andrievsky), because of the restricted observed spectral region and small number of available Fe II lines. We estimate an error in the gravity $\approx \pm 0.2$ dex. By varying the adopted $\log g$ value within the range \pm 0.2 dex, we obtained the iron abundance uncertainty of about 0.15 dex which is comparable with the typical error of abundance determination. The small gravity variations produce a similar uncertainty in the derived sodium abundance. The results of $\log g$ and $V_{\mathrm{t}}$ determinations are presented in Table 1.

\section{Elemental abundances}

\subsection{LTE calculations}

The LTE abundances of iron and sodium were derived from the equivalent widths (Table 2), using the Kurucz standard atmosphere models and the WIDTH9 code. The results on $\log \epsilon(\mathrm{Fe})$ and $\log \epsilon(\mathrm{Na})$ are given in Table 2 .

\subsection{NLTE results}

The NLTE abundances of $\mathrm{Na}$ in program stars were derived with the help of the modified version of the MULTI code (Carlsson 1986) described in Korotin et al. (1999) and Korotin et al. (1999).

The model of the sodium atom (proposed by Sakhibullin 1987) has been modified (see Korotin \& Mishenina 1999). The model consists of 27 levels of Na I and the ground level of NaII. The radiative transitions between the first 20 levels of $\mathrm{Na}$ I and the ground level of Na II were considered. Transitions between the rest levels were used only in the equations of particle number conservation. Finally, $46 b-b$ and $20 b-f$ transitions were included in the linearization procedure. For 34 transitions the radiative rates were fixed.

The NLTE calculations give abundance results (see Table 2) that differ from the LTE abundances in $\Delta$ value $\left(\Delta=\log \epsilon(\mathrm{Na}){ }_{\mathrm{NLTE}}-\log \epsilon(\mathrm{Na})_{\mathrm{LTE}}\right)$. In the last two columns we give the sodium abundances in program stars that are referred to the corresponding solar value and also normalized to the iron content. These results were used to investigate the dependence between the sodium overabundance and stellar gravity. 
Table 1. $T_{\text {eff }}$ determination for program stars.

\begin{tabular}{|c|c|c|c|c|c|c|c|c|c|c|}
\hline Star & $T(b-y)$ & $T(B-V)$ & $T(B 2-V 1)$ & $T(R-I)$ & $T(V-R)$ & $\sigma$ & $T_{\text {eff }}$ & $\log g$ & $V_{\mathrm{t}}$ & $\mathrm{Sp}$ \\
\hline HD 371 & 5250 & 5220 & & & & 15 & 5235 & 1.6 & 2.2 & G3 II \\
\hline HD 725 & 6375 & 6550 & & & & 88 & 6500 & 0.5 & 2.5 & F5 Ib-II \\
\hline HD 4362 & 5400 & 5440 & & & & 20 & 5420 & 1.5 & 3.7 & G0 Ib \\
\hline HD 5747 & 5120 & 5020 & & & & 50 & 5100 & 3.0 & 1.5 & G8 II \\
\hline HD 9900 & & 4430 & & & & & 4430 & 0.6 & 2.5 & G5 II \\
\hline HD 10806 & & & & & & & 5300 & 2.9 & 2.3 & G9 Ib \\
\hline HD 11544 & 5140 & 5130 & & & & 5 & 5135 & 0.7 & 2.9 & G2 Ib \\
\hline HD 15784 & 6500 & 6500 & & & & 0 & 6500 & 2.5 & 3.0 & F4 II \\
\hline HD 16901 & 5550 & 5530 & & & & 10 & 5540 & 1.1 & 3.5 & G0 Ib \\
\hline HD 20902 & 6175 & 6240 & 6160 & & & 25 & 6200 & 0.6 & 2.9 & F5 Ib \\
\hline HD 25291 & 6600 & 6690 & & & & 45 & 6750 & 1.0 & 3.0 & F0 II \\
\hline HD $134852^{*}$ & 6860 & 6690 & & & & 85 & 6750 & 2.4 & 3.4 & F2 II \\
\hline HD $139862^{*}$ & 5050 & 5040 & 4880 & 5080 & 4860 & 46 & 5000 & 2.0 & 1.7 & G8 II \\
\hline HD 159181 & 5250 & 5290 & 5140 & 5370 & 5460 & 54 & 5400 & 2.0 & 3.0 & G2 II \\
\hline HD $171635^{*}$ & 6000 & 5910 & 5820 & 5940 & 5720 & 49 & 6000 & 1.0 & 3.3 & F7 Ib \\
\hline HD $176155^{*}$ & 6400 & 6260 & 6000 & & & 117 & 6350 & 1.2 & 3.0 & F8 Ib \\
\hline HD 179784 & & 4915 & 4560 & 5150 & 4860 & 121 & 5100 & 1.8 & 2.8 & G5 Ib \\
\hline HD 180028 & 6000 & 6100 & & & & 50 & 6050 & 1.3 & 3.0 & F6 Ib \\
\hline HD $180583^{*}$ & 6360 & 6090 & 5940 & 6140 & 6140 & 67 & 6200 & 2.0 & 3.1 & F6 Ib-II \\
\hline HD 182296 & & 4930 & 4640 & 5060 & 4820 & 89 & 5000 & 1.0 & 2.8 & G3 Ib \\
\hline HD 182835 & 6550 & 6100 & 6480 & 6070 & & 125 & 6500 & 0.5 & 2.2 & $\mathrm{~F} 2 \mathrm{Ib}$ \\
\hline HD 185018 & 5750 & 5690 & 5530 & & & 66 & 5700 & 2.3 & 2.5 & G0 Ib \\
\hline HD 185758 & 5625 & 5640 & 5480 & 5660 & 5680 & 36 & 5500 & 3.6 & 2.6 & G0 II \\
\hline HD 185958 & 5100 & 4860 & 4730 & 5000 & 4850 & 64 & 5250 & 1.5 & 1.8 & G8 II \\
\hline HD 189671 & & & & & & & 4850 & 1.8 & 2.3 & G8 II \\
\hline HD 190323 & 5900 & 5760 & & & & 70 & 5900 & 0.1 & 4.2 & G0 Ia \\
\hline HD 190403 & & 4730 & & & & & 4850 & 1.5 & 2.5 & G5 Ib-II \\
\hline HD $193370^{*}$ & 6400 & 6410 & 6300 & 6220 & & 45 & 6350 & 1.5 & 3.2 & F5 Ib \\
\hline HD 194069 & & 5210 & 5030 & & & 90 & 5100 & 1.8 & 2.8 & G5 II \\
\hline HD 194093 & 6065 & 5920 & 5840 & 5960 & 6100 & 48 & 6000 & 0.6 & 3.5 & F8 Ib \\
\hline HD $194951^{*}$ & 6150 & 6290 & & & & 70 & 6350 & 1.0 & 2.8 & F1 II \\
\hline HD 195295 & 6500 & 6430 & 6360 & 6380 & & 31 & 6500 & 1.6 & 3.0 & F5 II \\
\hline HD 195593 & 6130 & 6260 & 6080 & 5640 & & 135 & 6100 & 0.5 & 2.6 & F5 Iab \\
\hline HD $199394^{*}$ & 5030 & 4950 & & & & 40 & 5000 & 2.2 & 1.4 & G5 II \\
\hline HD 200102 & 5350 & 5380 & 5110 & & & 85 & 5250 & 0.8 & 2.4 & G1 Ib \\
\hline HD 202109 & 5130 & 5070 & 4900 & 5120 & 5100 & 42 & 5100 & 2.5 & 1.5 & G8 II \\
\hline HD 202314 & 4750 & 4750 & 4640 & & 5070 & 93 & 4900 & 1.5 & 3.2 & $\mathrm{G} 2 \mathrm{Ib}$ \\
\hline HD $205603^{*}$ & 5200 & & & & & & 5200 & 3.1 & 1.2 & G8 II \\
\hline HD $206731^{*}$ & 5000 & 4990 & & & & 5 & 5000 & 2.0 & 1.8 & G8 II \\
\hline HD 206859 & 4670 & 4650 & 4540 & & 4550 & 34 & 4750 & 0.8 & 2.7 & G5 Ib \\
\hline HD $211153^{*}$ & 5125 & & & & & & 5125 & 2.5 & 1.4 & G8 Ib-II \\
\hline HD 216206 & 4950 & 5010 & 4780 & & & 69 & 5100 & 1.1 & 2.5 & G4 Ib \\
\hline HD 220102 & 6450 & 6580 & 6790 & & & 99 & 6600 & 1.0 & 2.7 & F5 II \\
\hline HD 220819 & & & & & & & 7000 & 0.5 & 1.3 & F0 II \\
\hline HD $221661^{*}$ & 4950 & 5060 & 4840 & & & 64 & 5000 & 2.3 & 1.5 & G8 II \\
\hline HD 223047 & 5180 & 5050 & 4840 & 5060 & 5120 & 58 & 5000 & 1.5 & 3.0 & G5 Ib \\
\hline HD 224165 & 4500 & 4660 & 4420 & & & 71 & 4600 & 0.5 & 2.3 & G8 Ib \\
\hline HD $225292^{*}$ & 5100 & 5070 & & & & 15 & 5100 & 2.7 & 1.3 & G8 II \\
\hline
\end{tabular}

* For stars denoted by asterisks, the reddenings were calculated. 
Table 2. Sodium line equivalent widths and abundance results.

\begin{tabular}{|c|c|c|c|c|c|c|c|c|c|}
\hline Star, HD & $6154 \AA$ & $6160 \AA$ & $\log \epsilon(\mathrm{Fe})$ & $\sigma$ & $\log \epsilon(\mathrm{Na})$ & $\sigma$ & $\Delta$ & {$[\mathrm{Na} / \mathrm{H}]$} & {$[\mathrm{Na} / \mathrm{Fe}]$} \\
\hline 371 & 68 & 99 & 7.41 & 0.10 & 6.35 & 0.03 & -0.09 & 0.10 & 0.19 \\
\hline 725 & 24 & 39 & 7.26 & 0.10 & 6.37 & 0.02 & -0.06 & 0.12 & 0.36 \\
\hline 4362 & 57 & 98 & 7.42 & 0.12 & 6.27 & 0.03 & -0.08 & 0.02 & 0.10 \\
\hline 5747 & 81 & 107 & 7.70 & 0.20 & 6.44 & 0.01 & -0.14 & 0.19 & -0.01 \\
\hline 9900 & 154 & 177 & 7.33 & 0.25 & 6.55 & 0.03 & -0.18 & 0.30 & 0.47 \\
\hline 10806 & 92 & 111 & 7.64 & 0.18 & 6.53 & 0.05 & -0.11 & 0.28 & 0.14 \\
\hline 11544 & 85 & 129 & 7.52 & 0.12 & 6.44 & 0.06 & -0.13 & 0.19 & 0.17 \\
\hline 15784 & 25 & 46 & 7.50 & 0.09 & 6.34 & 0.02 & -0.07 & 0.09 & 0.09 \\
\hline 16901 & 64 & 100 & 7.47 & 0.10 & 6.41 & 0.02 & -0.10 & 0.16 & 0.19 \\
\hline 20902 & 38 & 66 & 7.28 & 0.13 & 6.43 & 0.02 & -0.08 & 0.18 & 0.40 \\
\hline 25291 & 16 & 31 & 7.15 & 0.15 & 6.28 & 0.03 & -0.05 & 0.03 & 0.38 \\
\hline 134852 & 30 & 48 & 7.48 & 0.17 & 6.41 & 0.02 & -0.05 & 0.16 & 0.18 \\
\hline 139862 & 82 & 107 & 7.42 & 0.15 & 6.38 & 0.01 & -0.13 & 0.13 & 0.21 \\
\hline 159181 & 62 & 95 & 7.61 & 0.12 & 6.31 & 0.01 & -0.08 & 0.06 & -0.05 \\
\hline 171635 & 43 & 86 & 7.26 & 0.10 & 6.42 & 0.07 & -0.08 & 0.17 & 0.41 \\
\hline 176155 & 48 & 87 & 7.45 & 0.10 & 6.63 & 0.03 & -0.09 & 0.38 & 0.43 \\
\hline 179784 & 107 & 149 & 7.58 & 0.12 & 6.58 & 0.04 & -0.17 & 0.33 & 0.25 \\
\hline 180028 & 45 & 99 & 7.53 & 0.22 & 6.56 & 0.16 & -0.11 & 0.31 & 0.28 \\
\hline 180583 & 37 & 69 & 7.56 & 0.10 & 6.45 & 0.04 & -0.08 & 0.20 & 0.14 \\
\hline 182296 & 118 & 150 & 7.60 & 0.12 & 6.59 & 0.01 & -0.18 & 0.34 & 0.24 \\
\hline 182835 & 24 & 50 & 7.31 & 0.22 & 6.45 & 0.06 & -0.08 & 0.20 & 0.39 \\
\hline 185018 & 62 & 93 & 7.66 & 0.10 & 6.49 & 0.01 & -0.12 & 0.24 & 0.08 \\
\hline 185758 & 115 & 141 & 7.88 & 0.19 & 6.82 & 0.04 & -0.17 & 0.57 & 0.19 \\
\hline 185958 & 65 & 93 & 7.30 & 0.15 & 6.34 & 0.02 & -0.13 & 0.09 & 0.29 \\
\hline 189671 & 116 & 145 & 7.47 & 0.15 & 6.54 & 0.01 & -0.18 & 0.29 & 0.32 \\
\hline 190323 & 71 & 119 & 7.40 & 0.10 & 6.65 & 0.04 & -0.12 & 0.40 & 0.50 \\
\hline 190403 & 108 & 140 & 7.48 & 0.20 & 6.42 & 0.01 & -0.16 & 0.17 & 0.19 \\
\hline 193370 & 46 & 73 & 7.44 & 0.12 & 6.53 & 0.01 & -0.07 & 0.28 & 0.34 \\
\hline 194069 & 94 & 121 & 7.52 & 0.20 & 6.40 & 0.03 & -0.11 & 0.15 & 0.13 \\
\hline 194093 & 63 & 102 & 7.38 & 0.10 & 6.60 & 0.02 & -0.11 & 0.35 & 0.47 \\
\hline 194951 & 19 & 61 & 7.20 & 0.22 & 6.29 & 0.18 & -0.06 & 0.04 & 0.34 \\
\hline 195295 & 33 & 56 & 7.36 & 0.12 & 6.42 & 0.01 & -0.06 & 0.17 & 0.31 \\
\hline 195593 & 38 & 70 & 7.31 & 0.18 & 6.43 & 0.05 & -0.09 & 0.18 & 0.37 \\
\hline 199394 & 73 & 100 & 7.42 & 0.20 & 6.31 & 0.04 & -0.15 & 0.06 & 0.14 \\
\hline 200102 & 72 & 107 & 7.30 & 0.10 & 6.39 & 0.03 & -0.14 & 0.14 & 0.34 \\
\hline 202109 & 94 & 118 & 7.65 & 0.20 & 6.59 & 0.01 & -0.21 & 0.34 & 0.19 \\
\hline 202314 & 98 & - & 7.28 & 0.15 & 6.32 & - & -0.06 & 0.07 & 0.29 \\
\hline 205603 & 73 & 103 & 7.65 & 0.14 & 6.46 & 0.04 & -0.17 & 0.21 & 0.06 \\
\hline 206731 & 88 & 121 & 7.55 & 0.30 & 6.47 & 0.04 & -0.17 & 0.22 & 0.17 \\
\hline 206859 & 109 & 145 & 7.31 & 0.15 & 6.34 & 0.01 & -0.17 & 0.09 & 0.28 \\
\hline 211153 & 85 & 105 & 7.62 & 0.16 & 6.51 & 0.02 & -0.17 & 0.26 & 0.14 \\
\hline 216206 & 91 & 124 & 7.38 & 0.16 & 6.37 & 0.01 & -0.16 & 0.12 & 0.24 \\
\hline 220102 & 20 & 37 & 7.23 & 0.12 & 6.24 & 0.01 & -0.11 & 0.00 & 0.26 \\
\hline 220819 & 10 & 22 & 7.32 & 0.25 & 6.40 & 0.05 & -0.11 & 0.15 & 0.33 \\
\hline 221661 & 85 & 114 & 7.55 & 0.25 & 6.46 & 0.03 & -0.11 & 0.21 & 0.16 \\
\hline 223047 & 117 & 163 & 7.52 & 0.15 & 6.60 & 0.05 & -0.16 & 0.35 & 0.33 \\
\hline 224165 & 111 & 133 & 7.21 & 0.15 & 6.30 & 0.03 & -0.14 & 0.05 & 0.34 \\
\hline 225292 & 68 & 96 & 7.45 & 0.15 & 6.32 & 0.03 & -0.14 & 0.07 & 0.12 \\
\hline
\end{tabular}




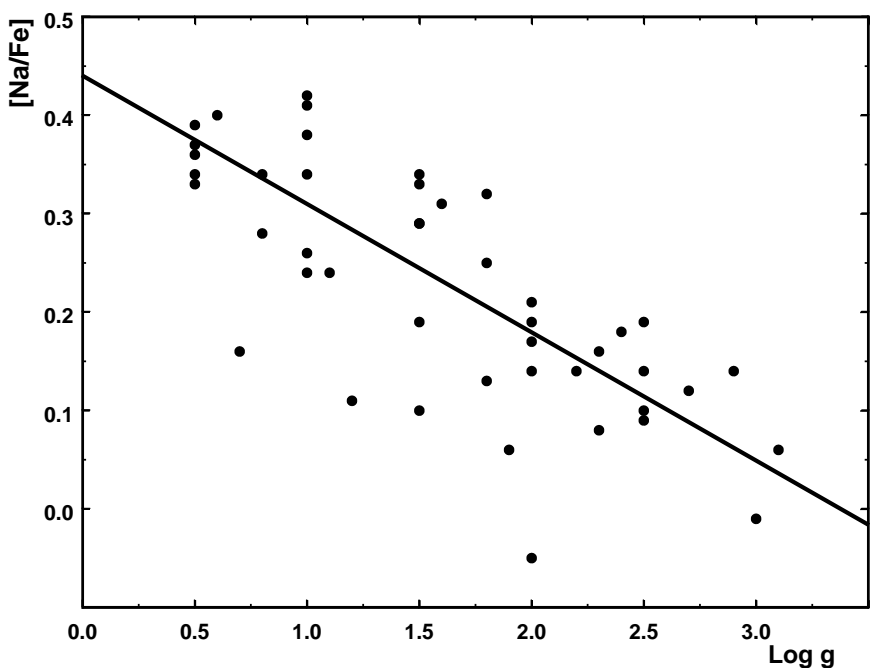

Fig. 1. Sodium abundance versus surface gravity.

\section{Sodium abundance vs. surface gravity}

In Fig. 1 we present graphically the main result of the present study. A clear dependence between the sodium abundance and stellar gravity has been detected:

$[\mathrm{Na} / \mathrm{Fe}]=-0.116( \pm 0.014) \log g+0.427( \pm 0.026)$.

As one can see from Fig. 1, no sodium overabundance is expected for the stars having gravities of about $1000 \mathrm{~cm} \mathrm{~s}^{-2}$. For the supergiant stars only a moderate overabundance of the sodium is seen.

A thorough discussion of the sodium overproduction in the low and intermediate mass AGB stars was recently presented by Mowlavi (1999a, 1999b), who calculated the surface sodium abundance prior to the thermally pulsing AGB phase (i.e. after 1st and $2 \mathrm{~d}$ dredge-up). According to his result, the surface sodium overabundace ranges from $\approx 0$ dex for the low mass stars to 0.25 dex for the intermediate mass stars at the evolutionary stages before the AGB phase (see Table 2 of that paper). Thus, our empirical data are in agreement with the results of those calculations, and they indicate that no extreme overabundances of the sodium are expected for the intermediate mass stars.

Of course, it would be much more astrophysically important to investigate a relation between the sodium abundance and stellar mass. Unfortunately it is quite difficult to estimate the correct masses of the stars of our sample, because we do not reliably know their luminosities. Without knowing this principal stellar parameter, we can state only qualitatively that the lower gravity stars are (on avarage) more luminous, and thus they should probably be more massive (the luminosities of supergiants are proportional to their masses, i.e. $L \sim M^{3.5}$, see e.g. Chiosi et al. 1992). This means that the detected $[\mathrm{Na} / \mathrm{Fe}]-\log g$ dependence implies the existence of $[\mathrm{Na} / \mathrm{Fe}]-$ mass relation, but its quantitative characteristics still should be evaluated.

Acknowledgements. SMA is grateful to Dr. F. Spite for help with the organizing of the observations. SMA would like to express his gratitude to the staff of the Haute Provence Observatory, and personally to Jean-Claude Mevohlon for provision of the excellent conditions during his observations. He also gratefully acknowledges the Ministère l'Enseignement Superieur et de la Recherche (France) for the possibility to perform part of this study at the Paris-Meudon Observatory and Haute-Provence Observatoire, FAPESP for the visiting professor fellowship (No. 2000/06587-3), and Instituto Astronômico e Geofisíco, Universidade de São Paulo for providing facility support. All necessary information about the program stars has been kindly provided by SIMBAD database operated at CDS (Strasbourg, France). The authors are grateful to the referee Dr. F. Spite for the detailed review of the manuscript and his useful comments.

\section{References}

Arellano Ferro, A., \& Parrao, L. 1990, A\&A, 239, 205

Boyarchuk, A., Hubeny, I., Kubat, J., Lyubimkov, L. S., \& Sakhibullin, N. A. 1988, Ap, 28, 202

Buser, R., \& Kurucz, R. L. 1992, A\&A, 264, 557

Carlsson, M. 1986, Uppsala Obs. Rep., 33

Castelli, F., Gratton, R. G., \& Kurucz, R. L. 1997, A\&A, 318, 841

Chiosi, C., Wood, P. R., Bertelli, G., Bressan, A., \& Mateo, M. 1992, ApJ, 385, 205

Denissenkov, P. A. 1990, Ap, 31, 588

El Eid, M. F., \& Champagne, A. E. 1995, ApJ, 451, 298

El Eid, M. F., \& Prantzos, N. 1993, Origin and Evolution of the Elements, ed. N. Prantzos, E. Vangioni-Flam, \& M. Casse (Cambridge Univ. Press), 279

Galazutdinov, G. A. 1992, Prepr. SAO RAS, No. 92

Korotin, S. A., Andrievsky, S. M., \& Luck, R. E. 1999, A\&A, 351,168

Korotin, S. A., Andrievsky, S. M., \& Kostynchuk, L. Yu. 1999, Ap\&SS, 260, 531

Korotin, S. A., \& Mishenina, T. V. 1999, ARep, 43, 533

Kovtyukh, V. V., \& Andrievsky, S. M. 1999, A\&A, 351, 597

Kunzli, M., North, P., Kurucz, R. L., \& Nicolet, B. 1997, A\&A, 122,51

Kurucz, R. L. 1991, Precision photometry: Astrophysics of the Galaxy, ed. A. G. D. Philip, A. R. Upgren, \& K. A. Janes (L. Davis Press), 1

Mowlavi, N. 1999, A\&A, 344, 617

Mowlavi, N. 1999, A\&A, 350, 73

Prantzos, N., Coc, A., \& Thibaud, J. P. 1991, ApJ, 379, 729

Sakhibullin, N. A. 1987, SvA, 31, 666

Sasselov, D. D. 1986, PASP, 98, 561

Takeda, Y., \& Takada-Hidai, M. 1994, PASJ, 46, 395 